\section{The Isotope Effect in the Spectrum of Silicon} Nitride.

RESULTs of a quantum theory analysis of the SiN bands and of the vibrational isotope effect in these bands were given in an earlier letter to NATURE (March 22, I924) and in a paper presented at a meeting of the American Physical Society (cf. Phys. Rev. $23,554,1924)$. It is now found that the equations given in the latter for these bands are incorrect. This is due to a wrong assignment of vibrational quantum numbers, corresponding to what may be described as an insidious violation of the combination principle. With the data first used, this violation was not apparent, but new data disclose systematic, although rather small, deviations. A new and, this time, correct assignment of quantum numbers has now been made. The following equation holds for the position of the null-lines of the $\mathrm{Si}^{28} \mathrm{~N}$ bands $\left(n^{\prime}=\right.$ vibrational quantum number of the initial, $n^{\prime \prime}$ that of the final state of the molecule):

$\nu=24234 \cdot 2+$ IOI $6 \cdot 30 n^{\prime}-17 \cdot 77 n^{\prime 2}+0.4 I^{\prime 3}$

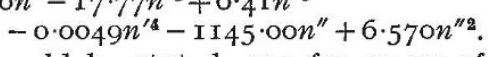

The null-lines, it should be stated, can for many of the bands be measured directly on the plates. At the low temperature of the active nitrogen used in generating the bands, the null-line appears as a conspicuous hole in the band structure, on the lowfrequency side of the head.

With the new numbering, the various apparent abnormalities previously noted disappear, and an analogy of the $\mathrm{SiN}$ bands to the violet $\mathrm{CN}$ bands is brought out. In particular, the isotope effect, previously thought abnormally large for the initial state of the molecule, is now completely normal. Agreement with the theory is exceedingly good if the emitter is assumed to be SiN. No other assumed emitter gives agreement with the experimental data; even for $\mathrm{SiO}$, the agreement is poor. Thus the value of the isotope effect in the identification of the emitters of band spectra, emphasised in a previous letter (April 5, I924), is again confirmed. As in the case of the BO bands, so in the case of SiN, the testimony of the isotope effect is backed up by the chemical evidence (NATURE, Sept. 6, I924, and Phys. Rev. 25, 259, (I925)).

The agreement of the results with theory is much better if the integral vibrational quantum numbers $0, I, 2, \ldots$. are assumed than if the half-integral numbers $\frac{1}{2}, I \frac{1}{2}, 2 \frac{1}{2}, \ldots$ are used. In this respect SiN differs from BO, for which the data indicate (cf. refs. last cited) that half-integral values are needed.

A detailed account of the work on the SiN bands is now being prepared for publication.

Jefferson Physical Laboratory,

Robert S. MULIIKEN, National Research Fellow. Harvard University, May 16.

Planetary Densities and Gravitational Pressure.

IN astronomical works the densities of the sun and planets are taken as the ratios of the masses to their apparent volume, the masses being determined by the periods of the bodies which revolve round them.

For the sun itself, and for several of the planets (notably for Jupiter and Saturn), the density so determined is much less than that of the earth, and it seems very improbable that this should really be the case, considering that the materials of which they are composed are the same as those which make up the earth, and that the gravitational pressure to which they are subjected is much greater than that which could be produced by terrestrial gravity.
In the case of the sun, Venus, Jupiter, and Saturn, in which the visible part is vapour or cloud, it seems most likely that there is a dense nucleus in which most of the mass is concentrated, and, assuming for the moment that the whole mass is so concentrated it is a simple and direct problem to determine the size of the nucleus for any given density.

The depth of the non-solid covering will be the difference between the apparent semidiameter and the radius of the nucleus, and if the latter has the density of the earth, the approximate dimensions in miles are as follows:

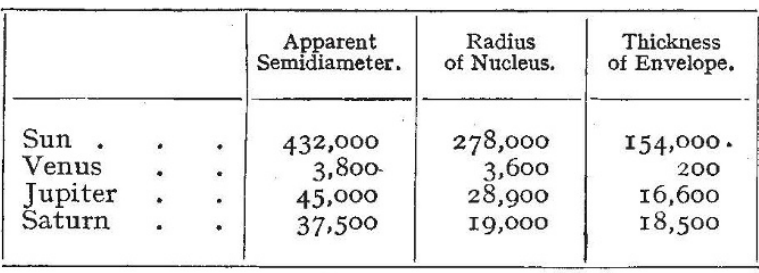

If the density depends on the gravitational pressure, the diameter of the nucleus will be less than the above for the sun, Jupiter, and Saturn, and rather greater for Venus.

There are no experiments on the variation of density of solids under large pressures. If a rod of the mean density of the earth is supposed to reach from the surface to the centre, the pressure on its base is just half that which the same mass would exert at the surface when resting on the same area. Taking the earth's radius as 2 I million feet and the earth's density as $5 \cdot 6$, this gives $I x, 400$ tons per square inch as the pressure at the earth's centre.

I have seen no mention of trustworthy experiments at even roo tons per square inch, and in my own work have never gone beyond 30 tons.

In some trials with precipitated chalk, using pressures of 25 tons to the inch, I have obtained blocks with a density of $x \cdot 6$ to $x \cdot 7$-much the same as the density of the upper chalk. In the lower chalk, however, the density sometimes exceeds 2 , and it would be interesting to know whether this is the result of gravitational pressure.

\section{A. Mallock.}

\section{Spiral Springs of Quartz.}

I AM greatly interested in the account in NATURE of June 20, p. 943, of the manufacture of spiral springs of fused quartz by Dr. Sliupas, and the comment by Prof. Boys on the achievement.

During the last eighteen months we have been employing similar springs of fused quartz in this laboratory in the measurement of sorption, a preliminary announcement of their use appearing in the Journal of the Am. Chem. Soc. for December of last year (Bakr and McBain, p. 2722), and a full account of the sorption balance is now being communicated.

Our experiences confirm in every way the observations made by Dr. Sliupas in his letter. Several members of the laboratory have prepared these springs of varying dimensions, using the ordinary coal-gas-air blow-flame for forming the coil. (The oxygen-gas flame was, of course, employed for the drawing-out of the fibres from the thick rods of quartz.) The quartz fibre is attached by a smear of sealing-wax to an ordinary arc-lamp carbon of suitable dimensions, which is supported in a well-bored cork, so that it may be rotated about its longitudinal axis. A small weight is suspended from the free end of the fibre, which is then coiled by slowly turning the carbon rod, the fibre being heated by the blow-flame at the point 